\title{
Syndecan-1 improves severe acute kidney injury prediction after pediatric cardiac surgery
}

\author{
Candice Torres de Melo Bezerra Cavalcante, MD, ${ }^{\text {a,b }}$ Klébia Magalhães Castelo Branco, MD, PhD, \\ Valdester Cavalcante Pinto Júnior, MD, ${ }^{\mathrm{a}}$ Gdayllon Cavalcante Meneses, MSc, \\ Fernanda Macedo de Oliveira Neves, MSc, ${ }^{b}$ Nayana Maria Gomes de Souza, MSc, ${ }^{a}$ \\ Kiarelle Lourenço Penaforte, MD, ${ }^{\mathrm{a}}$ Alice Maria Costa Martins, $\mathrm{PhD},{ }^{\mathrm{c}}$ and \\ Alexandre Braga Libório, $\mathrm{MD}, \mathrm{PhD}^{\mathrm{b}, \mathrm{d}, \mathrm{e}}$
}

\begin{abstract}
Objective: Acute kidney injury is a common occurrence after pediatric cardiac surgery and is associated with adverse patient outcomes. Syndecan-1 is a biomarker of endothelial glycocalyx damage, and its early increment after surgery can be associated with acute kidney injury.

Methods: We performed a prospective cohort study with 289 patients aged less than 18 years who underwent cardiac surgery at 1 reference institution. Postoperative plasma syndecan-1 was collected within the first 2 hours after cardiac surgery. Severe acute kidney injury, defined according to Kidney Disease: Improving Global Outcomes stage 2 or 3 , doubling of serum creatinine from the preoperative value, or need for dialysis during hospitalization, was the main outcome. Analyses were adjusted for clinical variables and "renal angina index" components (early decrease in estimated creatinine clearance from baseline and increase in percent of intensive care unit fluid overload on the first postoperative day).
\end{abstract}

Results: Plasma syndecan-1 levels measured early in the postoperative period were independently associated with severe acute kidney injury. The accuracy of postoperative syndecan-1 for the diagnosis of severe acute kidney injury was moderate (area under the curve receiver operating characteristic, 0.77; 95\% confidence interval, 0.68-0.85). The addition of syndecan-1 improved the discrimination capacity of a clinical model from 0.80 to $0.86(P=.004)$ and improved risk prediction, as measured by net reclassification improvement and integrated discrimination improvement. Postoperative sundecan-1 levels also were independently associated with longer length of intensive care unit and hospital stay.

Conclusions: Postoperative plasma syndecan-1 is associated with subsequent severe acute kidney injury and poor outcomes among children undergoing cardiac surgery. It may be useful to identify patients who are at increased risk for acute kidney injury after cardiac surgery. (J Thorac Cardiovasc Surg 2016;152:178-86)

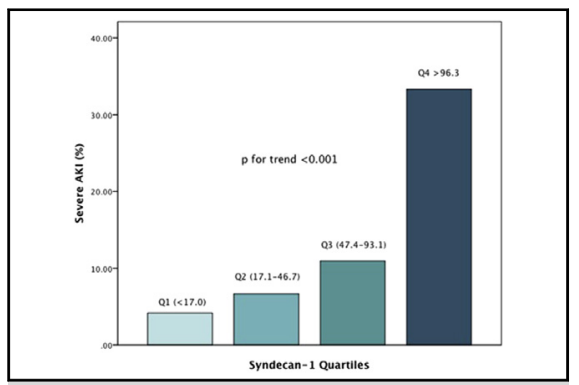

Quartiles of syndecan-1 had a graded relationship with the risk for severe AKI.

\section{Central Message}

Syndecan-1, a biomarker of endothelial glycocalyx damage, may be useful for risk stratifying children undergoing cardiac surgery.

\section{Perspective}

Postoperative syndecan-1 is associated with severe AKI in children who undergo cardiac surgery and adds value to risk stratification to predict severe AKI.

See Editorial Commentary page 187.
From the ${ }^{\mathrm{a} H o s p i t a l}$ do Coração de Messejana; ${ }^{\mathrm{b}}$ Medical Sciences Postgraduate Program, Department of Clinical Medicine, Universidade Federal do Ceará; ' Department of Clinical and Toxicological Analysis, Faculty of Pharmacy, Federal University of Ceara; ${ }^{\mathrm{d}}$ Universidade de Fortaleza-UNIFOR, Fortaleza, Ceara, Brazil; and ${ }^{\mathrm{e}}$ Instituto José Frota, Fortaleza, Ceará, Brazil.

Funded by the Conselho Nacional de Desenvolvimento Científico e Tecnológico (CNPq), Grant Number 470589/2014-0.

Received for publication Nov 30, 2015; revisions received March 9, 2016; accepted for publication March 27, 2016.

Address for reprints: Alexandre Braga Libório, MD, PhD, Avenida Abolição, 4043 Ap 1203, Fortaleza, Ceará, Brazil CEP 60165-082 (E-mail: alexandreliborio@ yahoo.com.br).

$0022-5223 / \$ 36.00$

Copyright $(2) 2016$ by The American Association for Thoracic Surgery

http://dx.doi.org/10.1016/j.jtcvs.2016.03.079
Acute kidney injury (AKI) is a common complication of cardiac surgery in both adults and children and is associated with mortality and poor outcomes. ${ }^{1,2}$ In children, AKI after cardiac surgery is associated with prolonged length of stay

Scanning this QR code will take you to supplemental figures, tables, and video for this article. 


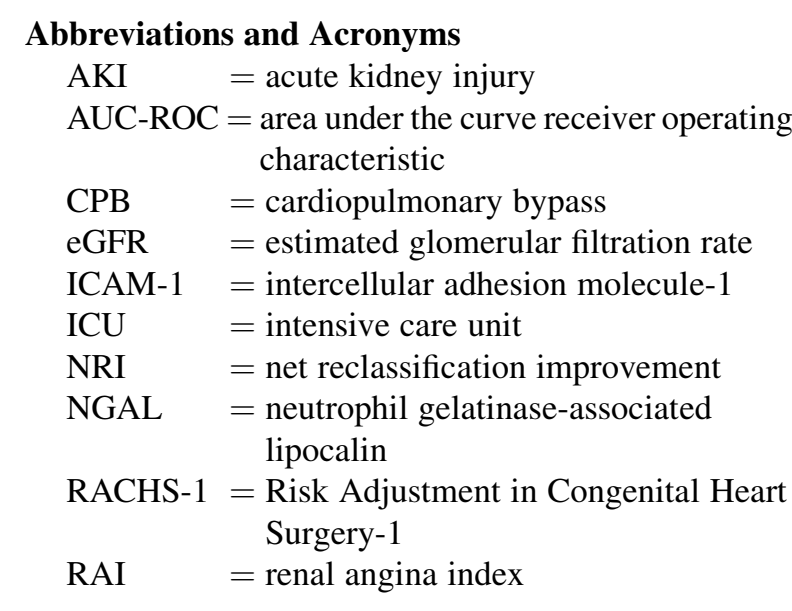

and mechanical ventilation. ${ }^{3}$ Also, in noncardiac pediatric intensive care unit (ICU) patients, AKI is independently associated with mortality. ${ }^{4}$ Serum creatinine, the traditional renal function marker, only increases appreciably after a $50 \%$ loss in glomerular filtration rate, impairing our ability to detect AKI early, thus missing the therapeutic window for mitigating AKI. ${ }^{5}$ This delay can explain, in part, the negative results in AKI therapeutic clinical trials.

Several studies have evaluated new biomarkers and their capacity to attain early AKI diagnosis in different clinical conditions. Most of these potential candidates are newly produced substances or markedly upregulated in kidney tissue under renal injury, being detected in serum or urine samples. Other biomarkers can emulate a systemic inflammation status detected in serum samples, such as interleukins 6,10 , and $18 .^{6}$

Endothelial injury is associated with activation of inflammation, structural changes leading to high expression of adhesion molecules, and procoagulation status. All of these situations are related to AKI pathophysiology. ${ }^{7}$ Endothelial glycocalyx is an approximately $1-\mu \mathrm{m}$-thick carbohydraterich structure that has antiadhesive and anticoagulant properties, protecting the endothelium and maintaining vascular barrier function. ${ }^{8}$ Syndecan-1, when measured in blood plasma, is a biomarker of endothelial glycocalyx damage. ${ }^{9}$

Cardiac surgery can damage endothelial glycocalyx in several ways: through cardiopulmonary bypass (CPB), by inducing a proinflammatory status, or by sympathetic activation. Considering that endothelial glycocalyx can have an important role in cardiac surgery-associated AKI pathophysiology, we hypothesized that syndecan-1 measured in the early postoperative period can provide additional benefits to predict AKI after pediatric cardiac surgery.

\section{MATERIALS AND METHODS}

\section{Study Design, Setting, and Patient Selection}

This was a prospective cohort study performed at a single center, Hospital do Coração de Messejana (Ceará, Brazil), a reference center for pediatric cardiac surgery for an estimated population of more than 6 million. Children aged 18 years or less undergoing cardiac surgery between September 2013 and December 2014 were eligible. Children receiving renal transplants or undergoing chronic dialysis were excluded. Children were recruited preoperatively and followed postoperatively. The institution's research ethics board approved the study. Informed consent was obtained from patients or parents/guardians before participation, with assent when appropriate.

\section{Data Collection and Study Procedures}

Demographic data and medical history were recorded. Preoperative estimated glomerular filtration rate (eGFR) was determined using the updated Schwartz equation, ${ }^{10}$ and eGFR percentiles were determined on the basis of published data on normal renal function of 651 children. ${ }^{11}$ Height was recorded to calculate preoperative eGFR. The following clinical variables were collected: age, gender, study site, CPB time, Risk Adjustment in Congenital Heart Surgery 1 (RACHS1) score $^{12}$ systolic blood pressure at ICU admission (in percentiles for sex and $\left.\operatorname{age}^{13}\right)$, maximum vasoactive inotropic score ${ }^{14}$ in the first 48 hours, lactate at ICU admission, fluid accumulation in the first 24 hours postoperatively in body weight percentage, and decline in preoperative eGFR using the first serum creatinine available after 24 hours postoperatively. RACHS- 1 contains 6 categories designed to differentiate surgical risk for mortality on the basis of the procedure. On ICU admission, additional blood was drawn for biomarkers (syndecan-1, e-selectin, and intercellular adhesion molecule-1 [ICAM-1]) measured within 2 hours after surgery and was concurrent with the first planned routine blood testing. Serum creatinine was measured routinely by the health care team, from shortly after surgery and every morning thereafter during ICU and hospital stay. Blood for biomarker measurement was centrifuged, and the plasma aliquots were stored at $-80^{\circ} \mathrm{C}$ until shipped for measurement. Each individual subject had preoperative and postoperative serum creatinine measured using the same assay at the same laboratory.

\section{Biomarker Measurements}

Syndecan-1 was measured as a biomarker of endothelial glycocalyx injury (Abcam, Cambridge, Mass). ${ }^{15}$ The detection range for syndecan1 is 8 to $256 \mathrm{ng} / \mathrm{mL}$, and the intra-assay coefficient of variation is $6.2 \%$. ICAM-1, a marker of endothelial cell activation, was measured using a commercially available enzyme-linked immunosorbent assay kit (Life Technologies Brasil, São Paulo, Brazil). Also, e-selectin, an endothelial cell adhesion molecule, was measured using a commercially available enzyme-linked immunosorbent assay kit (Abcam). Plasma neutrophil gelatinase-associated lipocalin (NGAL) was chosen as an example of renal biomarker, because it is one of the most often studied renal biomarkers after cardiac surgery. Plasma NGAL was measured using a commercially available enzyme-linked immunosorbent assay kit (Abcam).

\section{Outcomes}

Our primary outcome was severe AKI (stage 2/3). AKI outcomes were based on the Kidney Disease Improving Outcomes definition. ${ }^{16}$ Stage 1 AKI was a greater than $50 \%$ or $0.3 \mathrm{mg} / \mathrm{dL}$ (within 48 hours) serum creatinine increase from preoperative value, stage 2 was a 2 -fold increase in serum creatinine, and stage 3 was a 3 -fold increase in serum creatinine or patients starting dialysis during hospitalization. Secondary outcomes were length of ICU and hospital stay, mechanical ventilation duration, and hospital mortality.

\section{Statistical Analysis}

Continuous variables were compared using a 2-sample $t$ test or MannWhitney test, and dichotomous variables were compared with chi-square or 
Fisher exact test. Post hoc test after the chi-square test was performed when more than 2 categories were present. ${ }^{17}$ Correlations were performed using Spearman's rank correlation. To evaluate the association of biomarkers with AKI, we divided the cohort into quintiles on the basis of the postoperative syndecan-1 measurement. Unadjusted trends across biomarker quintiles were assessed by the Cochran-Armitage test for dichotomous outcomes and the Jonckheere-Terpstra test for continuous outcomes. Adjusted trends were assessed using contrasts in linear or logistic regression depending on the outcome (Wald chi-square test). To evaluate the association between biomarkers and AKI, logistic regression models were used. We used the model adjusting for important covariates that predict AKI in the pediatric cardiac surgery setting: The first model included age, gender, baseline eGFR, surgical complexity, and use and duration of CPB. Moreover, we included the components of the newly described renal angina index (RAI): early decrease in estimated creatinine clearance from baseline and increase in percent of ICU fluid overload on the first postoperative day. ${ }^{18}$ These variables also were used to construct a clinical model to predict severe AKI. To avoid overfitting of the model, we used penalized maximum likelihood estimation, ${ }^{19}$ which yielded shrunk regression coefficients. The optimum penalty factor that maximized the modified Akaike information criterion was used.

Area under the curve receiver operating characteristic (AUC-ROC) values were calculated for biomarkers and the clinical model. For syndecan-1, the optimal point was defined according to the highest Youden index, which was calculated as $[1-(1-$ sensitivity $)+(1-$ specificity $)]$. After that, syndecan-1 was added to the clinical model and the AUC-ROC values were compared using DeLong and colleagues' method. ${ }^{20}$ Furthermore, to evaluate the effect of biomarkers on AKI risk prediction, the net reclassification improvement (NRI) and integrated discrimination improvement were determined. ${ }^{21,22}$ When comparing the clinical model with the biomarker, an improvement in reclassification is defined when the risk of a patient with AKI is reclassified to a higher-risk category or a patient without AKI is reclassified to a lower-risk category. For the NRI analysis of the prediction of severe AKI, risk categories were defined as low $(<10 \%)$, medium $(10 \%-25 \%)$, or high $(>25 \%)$ on the basis of the clinical model. ${ }^{23}$

Likewise, a worse reclassification occurs if a patient with AKI moves down a risk category or a patient without AKI moves up a risk category. NRI is the difference in the proportion of improvements in reclassification and the proportion of worse reclassifications $\left(\mathrm{NRI}=\left(\mathrm{p}_{\mathrm{up}} \mathrm{AKI}-\right.\right.$ $\left.\mathrm{p}_{\text {down }} \mathrm{AKI}\right)+\left(\mathrm{p}_{\text {down }}\right.$ non-AKI - $\mathrm{p}_{\text {up }}$ non-AKI $)$, where $\mathrm{p}_{\text {up }} \mathrm{AKI}$ is the number of patients with AKI moving up divided by the number of patients with AKI, $p_{\text {down }} \mathrm{AKI}$ is the number of patients with AKI moving down divided by the number of patients with AKI, $p_{u p}$ non-AKI is the number of patients without AKI moving up divided by the number of patients without AKI, and $\mathrm{p}_{\text {down }}$ non-AKI is the number of patients without AKI moving down divided by the number of patients without AKI). Analysis of the data was performed using SPSS 19.0 for Windows (SPSS Inc, Chicago, Ill) and R version 2.14.1 (R Development Core Team, Vienna, Austria).

\section{RESULTS}

\section{Characteristics of the Study Cohort}

A total of 295 participants were included, but 6 patients were excluded because of withdrawal of informed consent $(\mathrm{n}=1)$ and lack of blood samples $(\mathrm{n}=5)$. In the final analysis, 289 patients were included $(62.0 \%$ of the patients were aged $<2$ years, and $52.7 \%$ were female). The majority of patients $(72 \%)$ underwent $\mathrm{CPB}$. The mean preoperative eGFR was $92.7 \mathrm{~mL} / \mathrm{min} / 1.73 \mathrm{~m}^{2}$. In the 7 days after cardiac surgery, 37 patients $(12.8 \%)$ developed severe AKI; 19 patients $(6.6 \%)$ received acute dialysis, and 22 patients (7.6\%) died before hospital discharge. Patients who developed severe AKI had higher RACHS-1 score, longer CPB time, and lower systolic blood pressure at ICU admission. For RAI criteria, severe AKI was associated with high fluid accumulation and eGFR decline on the first postoperative day (Table 1). The majority of patients had severe AKI diagnosed up to day 3 after surgery $(\mathrm{n}=35,94.6 \%)$.

\section{Association of Syndecan-1 With Clinical and Surgical Variables}

Postoperative syndecan-1 value ( 0 - to 2-hour period) after ICU admission had a small association with preoperative eGFR $(r=-0.145, P=.016)$ and systolic blood pressure at ICU admission $(r=-0.144, P=.018)$, and only a trend toward fluid accumulation on the first postoperative day $(\mathrm{r}=0.107, P=.070)$. There was no association with time of $\mathrm{CPB}$ or the other measured endothelial biomarkers (e-selectin, ICAM-1, and plasma NGAL).

\section{Association of Postoperative Syndecan-1 and Severe Acute Kidney Injury}

Median postoperative syndecan-1 levels were higher in patients with severe AKI (103.6 [interquartile range, 61.2-228.7] vs 42.3 [interquartile range, 15.3-78.9], $P<.001)$. Associations between the postoperative syndecan-1 levels, categorized into quartiles, and the risk of AKI are shown in Figure 1. Quartiles of syndecan-1 showed a graded association with the risk for severe AKI, ranging from $4.2 \%$ to $30.6 \%$ ( $P$ value for trend $<.001)$. After adjustment for clinical variables, the fourth quartile was significantly associated with severe AKI (Table 2). There was no difference regarding ICAM-1 or e-selectin levels in children with or without severe AKI (Figure E1).

\section{Diagnostic Testing}

The AUC-ROCs for postoperative syndecan-1, ICAM-1, and e-selectin are shown in Figure 2. Although ICAM-1 and e-selectin showed no discrimination value for severe AKI prediction, syndecan-1 had an AUC of 0.77. Syndecan-1 threshold value with maximal sensitivity and specificity was $66.4 \mathrm{ng} / \mathrm{mL}$ (sensitivity of $75.7 \%$ and specificity of $69.1 \%)$.

\section{Added Benefit of Postoperative Syndecan-1 to Predict Severe Acute Kidney Injury Above Clinical and Biomarker Prediction}

The clinical prediction model with the preoperative and intraoperative variables (including RAI variables) for AKI had an AUC of 0.81. Adding postoperative syndecan-1 as a biomarker to the clinical model improved discrimination of 0.87 ( $P=.003$ for AUC-ROC comparison) (Figure 3). 
TABLE 1. Cohort description of children according to severe acute kidney injury status

\begin{tabular}{|c|c|c|c|c|}
\hline & $\begin{array}{l}\text { All patients } \\
(\mathbf{n}=\mathbf{2 8 9})\end{array}$ & $\begin{array}{l}\text { No severe AKI } \\
\quad(\mathbf{n}=\mathbf{2 5 2})\end{array}$ & $\begin{array}{c}\text { Severe AKI } \\
\quad(\mathbf{n}=\mathbf{3 7})\end{array}$ & $P$ \\
\hline \multicolumn{5}{|l|}{ Age at time of surgery, $\mathrm{n}(\%)$} \\
\hline$<1 \mathrm{mo}$ & $36(12.4)$ & $29(11.5)$ & $7(18.9)$ & ns \\
\hline $1 \mathrm{mo}$ to $2 \mathrm{y}$ & $143(49.5)$ & $123(48.8)$ & $20(54.1)$ & ns \\
\hline $2-13$ y & $90(31.1)$ & $82(32.5)$ & $8(21.6)$ & ns \\
\hline $13-18$ y & $20(6.9)$ & $18(7.1)$ & $2(5.4)$ & ns \\
\hline Male gender, $\mathrm{n}(\%)$ & $137(47.3)$ & $120(47.6)$ & $17(45.9)$ & .86 \\
\hline \multicolumn{5}{|l|}{ Renal function } \\
\hline Preoperative eGFR $\left(\mathrm{mL} / \mathrm{min} / 1.73 \mathrm{~m}^{2}\right)$, mean $\pm \mathrm{SD}$ & $93 \pm 72$ & $92 \pm 76$ & $99 \pm 71$ & .65 \\
\hline \multicolumn{5}{|l|}{ RACHS-1 category, n (\%) } \\
\hline 1 & $48(16.6)$ & $48(19.0)$ & - & $<.05$ \\
\hline 2 & $94(32.5)$ & $87(34.5)$ & $7(18.9)$ & $<.05$ \\
\hline 3 & $107(37.0)$ & $86(34.1)$ & $21(56.8)$ & $<.05$ \\
\hline 4 & $38(13.1)$ & $29(11.5)$ & $9(24.3)$ & ns \\
\hline Not categorized & $2(0.8)$ & $2(0.9)$ & & ns \\
\hline CPB use, $\mathrm{n}(\%)$ & $208(72.0)$ & $178(70.6)$ & $30(81.1)$ & \\
\hline $\mathrm{CPB}$ time (min), mean $\pm \mathrm{SD}$ & $75 \pm 39$ & $71 \pm 36$ & $98 \pm 47$ & $<.01$ \\
\hline Crossclamp time (min), mean $\pm \mathrm{SD}$ & $45 \pm 31$ & $43 \pm 30$ & $55 \pm 39$ & $<.01$ \\
\hline Systolic blood pressure at ICU admission (percentile for sex and age), mean \pm SD & $38 \pm 12$ & $40 \pm 12$ & $35 \pm 12$ & .03 \\
\hline Serum lactate at ICU admission $(\mathrm{mmol} / \mathrm{L})$, mean $\pm \mathrm{SD}$ & $2.6 \pm 1.1$ & $2.4 \pm 0.9$ & $4.6 \pm 3.3$ & $<.01$ \\
\hline Maximum VIS, median (IQR) & $0(0-7)$ & $0(0-6)$ & $9(0-24)$ & $<.01$ \\
\hline Fluid accumulation in the first $24 \mathrm{~h}(\%$ of $\mathrm{BW})$, mean $\pm \mathrm{SD}$ & $1.1 \pm 3.5$ & $0.8 \pm 3.1$ & $3.1 \pm 4.9$ & $<.01$ \\
\hline \multicolumn{5}{|l|}{ Outcomes } \\
\hline Time on mechanical ventilation (d), median (IQR) & $1(1-5)$ & $1(1-3)$ & $9(2-26)$ & $<.01$ \\
\hline Length of ICU stay (d), median (IQR) & $5(2-13)$ & $4(2-10)$ & $19(7-40)$ & $<.01$ \\
\hline Length of hospital stay (d), median (IQR) & $15(9-28)$ & $14(9-25)$ & $32(20-64)$ & $<.01$ \\
\hline Dialysis, n (\%) & $19(6.6)$ & - & $19(51.3)$ & - \\
\hline Mortality, n (\%) & $22(7.6)$ & $11(4.6)$ & $11(30.6)$ & $<.01$ \\
\hline
\end{tabular}

Severe AKI is defined as the need for dialysis or a 2-fold increase in serum creatinine during hospitalization. Percentile eGFR was calculated by quantile regression based on published normal renal function measured by nuclear medicine scan glomerular filtration rate in 651 children (ref). The RACHS-1 consensus-based score system categorizes the complexity of surgery. Uncategorized RACHS-1 scores were not included in the continuous summary of RACHS-1 scores. AKI, Acute kidney injury; $S D$, standard deviation; $n s$, not significant; $e G F R$, estimated glomerular filtration rate; $R A C H S$ - 1 , Risk Adjustment in Congenital Heart Surgery-1; $C P B$, cardiopulmonary bypass; $I C U$, intensive care unit; $V I S$, vasoactive inotropic score; $I Q R$, interquartile range; $B W$, body weight.

Postoperative syndecan-1 also improved the classification accuracy of AKI prediction. The NRI resulting by biomarker inclusion was amplified by both reclassification of nonevents (ie, patients without severe AKI - NRI 0.08 ) and event (ie, patients with severe AKI - NRI 0.13 ). The overall NRI was 0.21 . For comparison, we also incorporated plasma NGAL into the clinical model, and there was no additional improvement (AUC-ROC, 0.81 ). The NRI resulting by plasma NGAL inclusion was 0.01 in the nonevent group and 0.02 in the event group. A complete summary of diagnostic tests is shown in Table 3.

\section{Sensitivity Analysis}

A summary of the main results of the analysis of these subgroups is shown in Tables E1 to E4. Because several studies improved their cohorts by adding children undergoing RACHS-1 2 or more surgeries and excluded infants aged less than 1 month, we also performed a sensitivity analysis with this subpopulation $(\mathrm{n}=203)$ and another analysis with only patients undergoing aortic crossclamping $(\mathrm{n}=189)$. In these subpopulations, postoperative syndecan-1 remained independently associated with AKI (Tables E1 and E3).

\section{Syndecan-1 and Nonrenal Outcomes}

Postoperative syndecan-1 was linearly associated with longer length of ICU and hospital stay after adjustment for other prognostic factors. In relation to length of mechanical ventilation, there was a trend for association with quartiles of syndecan-1 in the multivariate analysis (Figure 4).

\section{DISCUSSION}

In this study, we evaluated 3 endothelial biomarkers regarding their capacity to predict severe AKI after pediatric cardiac surgery. Although plasma ICAM-1 and e-selectin (markers of endothelial cell activation) were 


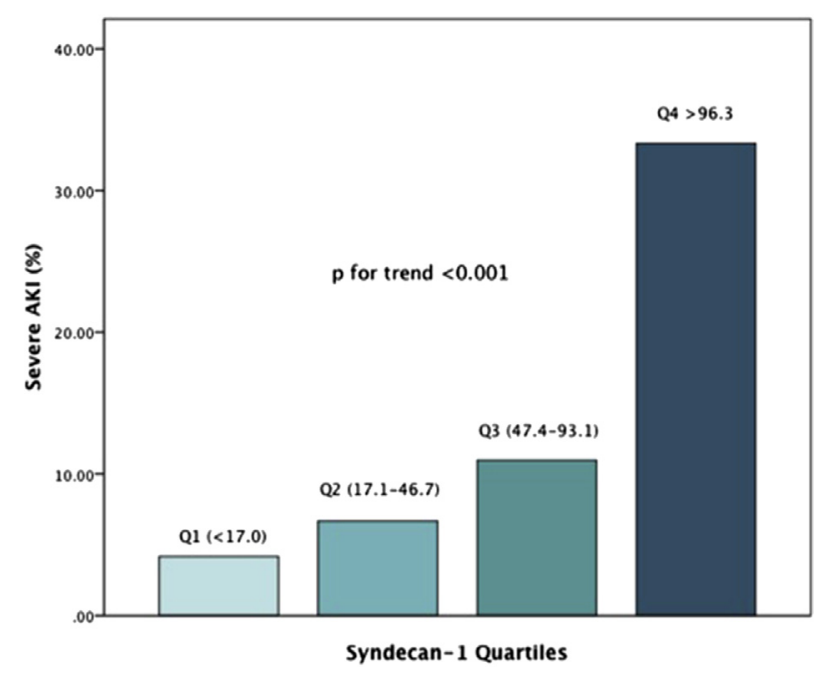

FIGURE 1. Quartiles of the first postoperative syndecan-1 level had a graded relationship with the risk for severe AKI. AKI, Acute kidney injury.

not increased in children with severe AKI, syndecan-1 was strongly and independently associated with severe AKI after cardiac surgery. Moreover, when added to a clinical model that included, in addition to other traditional clinical variables, RAI components, syndecan-1 discriminated patients who did or did not develop severe AKI.

Endothelial glycocalyx acts as a competent permeability barrier and an antiadhesive interface with blood. Disruption of this structure has been shown to increase capillary permeability, attach leucocytes and platelets, leading to tissue edema and accentuated inflammation, and increase procoagulant state. ${ }^{8}$ Syndecan-1 is a marker of endothelial glycocalyx damage that is increased after cardiac surgery. ${ }^{24,25}$ Although syndecan-1 is not a renal-specific biomarker, there has been recent increasing evidence that endothelial injury has an important role in AKI pathophysiology. ${ }^{7}$ Other nonrenalspecific biomarkers have been tested for AKI prediction

TABLE 2. Association of postoperative syndecan-1 and severe acute kidney injury

\begin{tabular}{|c|c|c|}
\hline \multirow{2}{*}{$\begin{array}{c}\text { Syndecan } \\
\text { quartiles } \\
\text { (cutoffs in } \mathbf{n g} / \mathbf{m L} \text { ) }\end{array}$} & \multicolumn{2}{|c|}{ Severe AKI* } \\
\hline & $\begin{array}{c}\text { Crude OR } \\
(95 \% \text { CI })\end{array}$ & $\begin{array}{c}\text { Adjusted OR } \\
\text { full } \uparrow(95 \% \text { CI })\end{array}$ \\
\hline Q1 $(<17.0)$ & 1 (reference) & 1 (reference) \\
\hline Q2 (17.1-46.7) & $1.64(0.38-7.14)$ & $1.42(0.29-7.00)$ \\
\hline Q3 (47.4-93.1) & $2.83(0.72-11.13)$ & $2.05(0.45-9.29)$ \\
\hline Q4 (>96.3) & $11.50(3.29-40.20)$ & $8.87(2.31-34.03)$ \\
\hline
\end{tabular}

$A K I$, Acute kidney injury; $O R$, odds ratio; $C I$, confidence interval. *Severe AKI is defined as need for dialysis or 2-fold increase in serum creatinine during hospitalization. $\dagger$ Adjusted for age (per year), gender, CPB time 120 min, RACHS- $1 \geq 3$, maximum vasoactive inotropic score in the first $48 \mathrm{~h}$, lactate at ICU admission preoperative eGFR percentile, decrease in estimated creatinine clearance from baseline on the first postoperative day, and increase in percent of ICU fluid overload on the first postoperative day.

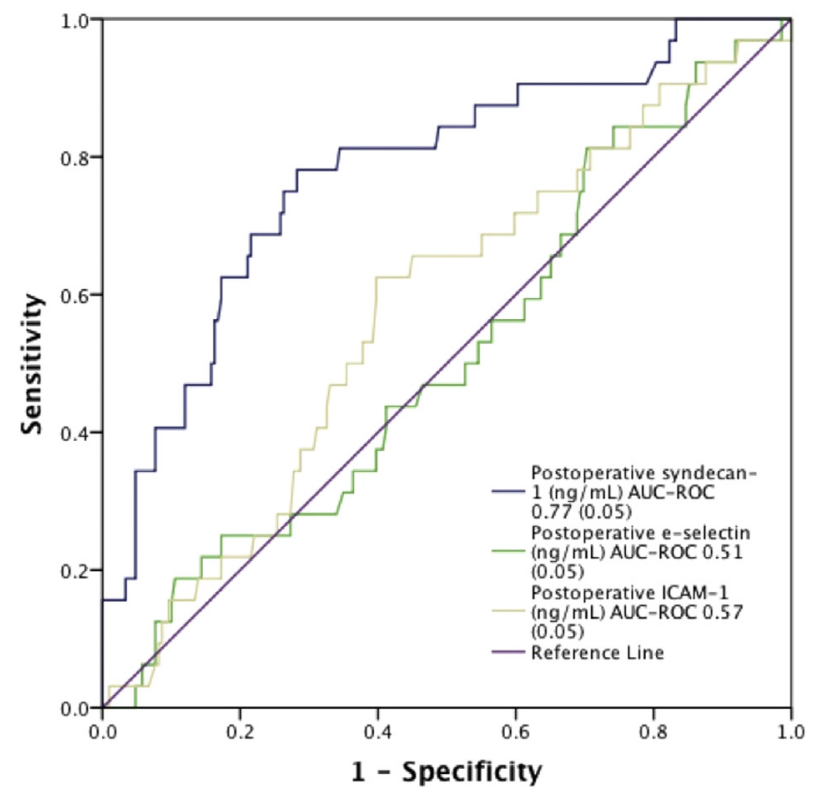

FIGURE 2. Diagnostic performance of the early postoperative value of plasma syndecan-1, ICAM-1, and e-selectin for the detection of severe AKI. AUC-ROC, Area under the curve receiver operating characteristic; ICAM-1, intercellular adhesion molecule-1.

with variable results. ${ }^{26,27}$ We previously showed that syndecan-1 can predict AKI in patients with heart failure $^{28}$ and is associated with leptospirosis-related AKI. ${ }^{29}$

Previous studies have evaluated syndecan-1 increment in the course of cardiac surgery with and without CPB. In adults and children, peak values of syndecan-1 were achieved early after declamping. ${ }^{25,30}$ This well-known early increment in syndecan-1 levels led us to choose an early point in time in the postoperative period (02 hours). In patients with chronic kidney disease, syndecan-1 is increased, ${ }^{31}$ making it a possible bias. In our data, syndecan-1 was only weakly inversely associated with preoperative renal function. This weak association can be explained because children had only minimal eGFR variation around the normal range. Another possible bias in relation to syndecan-1 use as a biomarker in patients undergoing cardiac surgery was the possible collinearity with CPB duration, a hypothesis not confirmed in our data.

In comparison with other renal and cardiac biomarkers evaluated in the perioperative period of cardiac surgery in children, syndecan- 1 alone showed at least a similar performance when compared with other renal biomarkers $^{23,32-34}$ and was superior to cardiac biomarkers ${ }^{27}$ in predicting renal events. In a study evaluating several different renal biomarkers, the best AUC-ROC achieved in the early postoperative period was the one found for urine interleukin-18 (AUC-ROC, 0.72). ${ }^{23}$ In another 


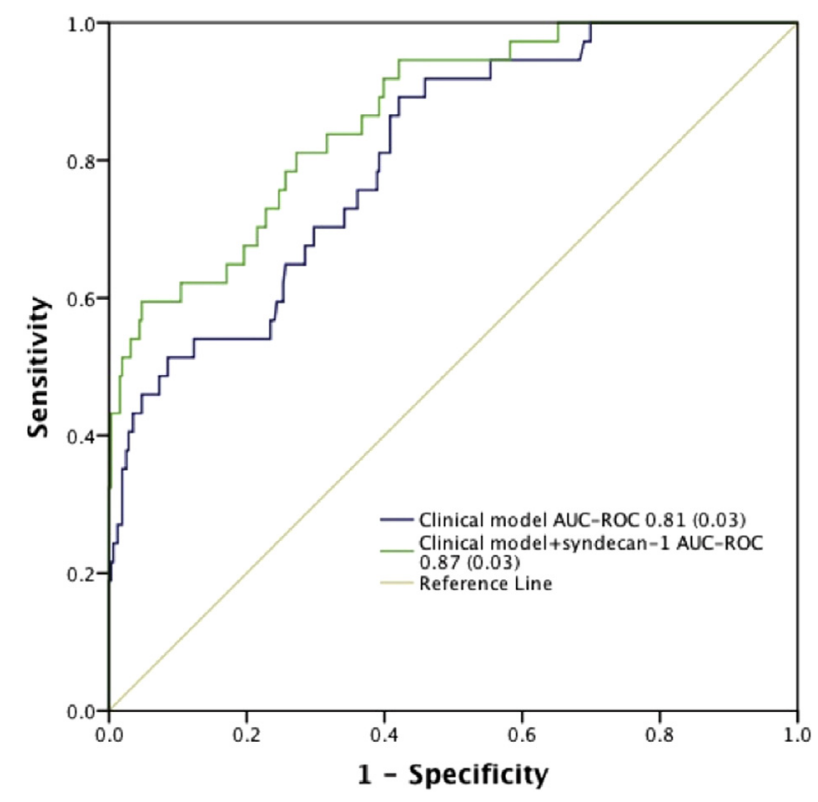

FIGURE 3. Diagnostic performance of a clinical model including age, sex, CPB use, CPB time more than 120 minutes, RACHS-1 3 or more, preoperative eGFR percentile, systolic blood pressure at ICU admission (percentile), maximum vasoactive inotropic score in the first 48 hours, lactate at ICU admission, and "renal angina index" components (early decrease in estimated creatinine clearance from baseline and increase in percent of ICU fluid overload on the first postoperative day) adding or not adding the early postoperative value of plasma syndecan-1. AUC$R O C$, Area under the curve receiver operating characteristic.

study, urinary cystatin-C had an AUR-ROC to predict AKI of 0.80 when measured early in the postoperative period. ${ }^{34} \mathrm{~A}$ recent study evaluated cardiac biomarkers in the preoperative and postoperative periods to predict AKI, and heart-type fatty acid binding protein had the best AUC-ROC when added to a clinical model (AUCROC, 0.80). ${ }^{27}$

We also evaluated a clinical model that included variables already used in previous studies, as well as variables included in the recently described RAI. ${ }^{18}$ Although RAI was described for critically ill children, we used its components; we considered all postoperative patients in the same group risk and included early changes in eGFR and fluid overload. By adding these variables, we achieved a good performance with the clinical model (for comparison, in the TRIBE-AKI consortium, the clinical model had an AUC-ROC of 0.75). Although great changes in the AUC-ROC values are not expected when comparing nested models, mainly when the first model had already achieved a good AUC-ROC, syndecan-1 yet increased the discrimination performance of this extended clinical model.

Unlike renal biomarkers, plasma syndecan-1 increase occurs regardless of tubular injury. Therefore, we hypothesized that adding 1 renal tissue biomarker could provide substantial additional predictive information. To evaluate this hypothesis, we also measured plasma NGAL. Although there was no difference in performance when NGAL was added to the clinical/syndecan- 1 model, this result must be considered with caution. Although plasma NGAL was chosen because it is one of the most often studied renal biomarkers, ${ }^{35}$ in our study it showed only a moderate AUC-ROC, adding only marginal benefit to the clinical model. It is possible that other renal biomarkers can be of value when used in combination with syndecan-1.

In addition to the potential use of postoperative syndecan-1 in risk stratification for severe AKI after pediatric cardiac surgery, our study also highlights the importance of glycocalyx damage in the pathophysiology of cardiac surgery-associated AKI. Maintenance of endothelial glycocalyx integrity can be a therapeutic target to reduce AKI in this setting. Other measured endothelial biomarkers (ICAM-1 and e-selectin) were not associated with AKI in our study. Our data are in accordance with a previous study that failed to demonstrate any difference regarding these adhesion molecules in patients with sepsis who did or did

TABLE 3. Diagnostic performance and categoric net reclassification improvement of clinical model adding or not adding postoperative biomarker

\begin{tabular}{|c|c|c|c|c|c|c|c|c|}
\hline & \multicolumn{8}{|c|}{ Severe AKI } \\
\hline & $\begin{array}{c}\text { AUC-ROC } \\
(95 \% \text { CI })\end{array}$ & $\begin{array}{c}P \text { for } \\
\text { comparison }\end{array}$ & $\begin{array}{l}\text { Nonevent } \\
\text { NRI (SE) }\end{array}$ & $\begin{array}{c}\text { Event } \\
\text { NRI (SE) }\end{array}$ & $\begin{array}{l}\text { Total } \\
\text { NRI }\end{array}$ & $\begin{array}{l}\text { Nonevent } \\
\text { IDI (SE) }\end{array}$ & $\begin{array}{c}\text { Event } \\
\text { IDI (SE) }\end{array}$ & $\begin{array}{l}\text { Total } \\
\text { IDI }\end{array}$ \\
\hline Plasma NGAL (ng/mL) & $0.67(0.58-0.77)$ & - & - & - & - & & & \\
\hline Plasma syndecan-1 & $0.77(0.68-0.85)$ & - & - & - & - & & & \\
\hline Clinical model & $0.81(0.74-0.88)$ & - & - & - & - & & & \\
\hline Clinical model + NGAL & $0.81(0.74-0.86)$ & $.319^{*}$ & $0.01(0.01)^{*}$ & $0.02(0.02)^{*}$ & $0.03^{*}$ & $<0.01(0.01)^{*}$ & $0.01(0.01)^{*}$ & $0.012^{*}$ \\
\hline Clinical model + syndecan-1 & $0.87(0.79-0.92)$ & $<.05 \dagger$ & $0.08(0.02)^{*}$ & $0.13(0.05)^{*}$ & $0.21 *$ & $0.03(0.01)^{*}$ & $0.06(0.01)^{*}$ & $0.09 *$ \\
\hline Clinical model + NGAL + & $0.87(0.79-0.91)$ & $.651 \dagger$ & $-0.01(0.01) \ddagger$ & $0.03(0.03) \ddagger$ & $0.02 \ddagger$ & $-0.01(0.01) \ddagger$ & $0.01(0.01) \ddagger$ & $0.01 \ddagger$ \\
\hline
\end{tabular}

syndecan-1

Clinical model: age, sex, CPB use, CPB time $>120$ minutes, RACHS-1 $\geq 3$, preoperative eGFR percentile, systolic blood pressure at ICU admission (percentile) and "renal angina index" components (early decrease in estimated creatinine clearance from baseline and increase in percent ICU fluid overload on the first postoperative day). AKI, Acute kidney injury; $A U C-R O C$, area under the curve receiver operating characteristic; $N R I$, net reclassification improvement; $S E$, standard error; $I D I$, integrated discrimination improvement; $N G A L$, neutrophil gelatinase-associated lipocalin. *Versus clinical model. †Versus clinical model and clinical model + NGAL. $\ddagger$ Versus clinical model + syndecan-1. 
Length of Hospital Stay

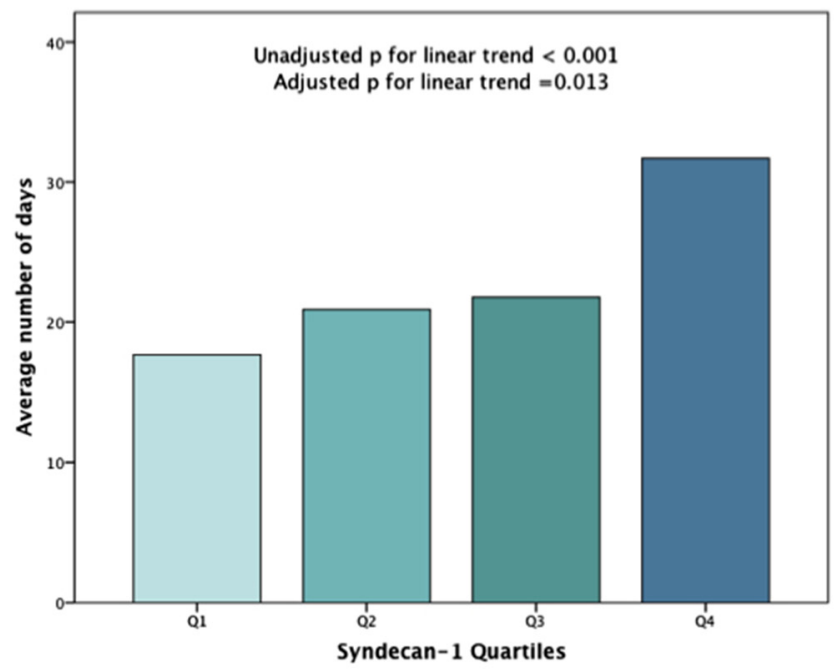

Length of ICU Stay

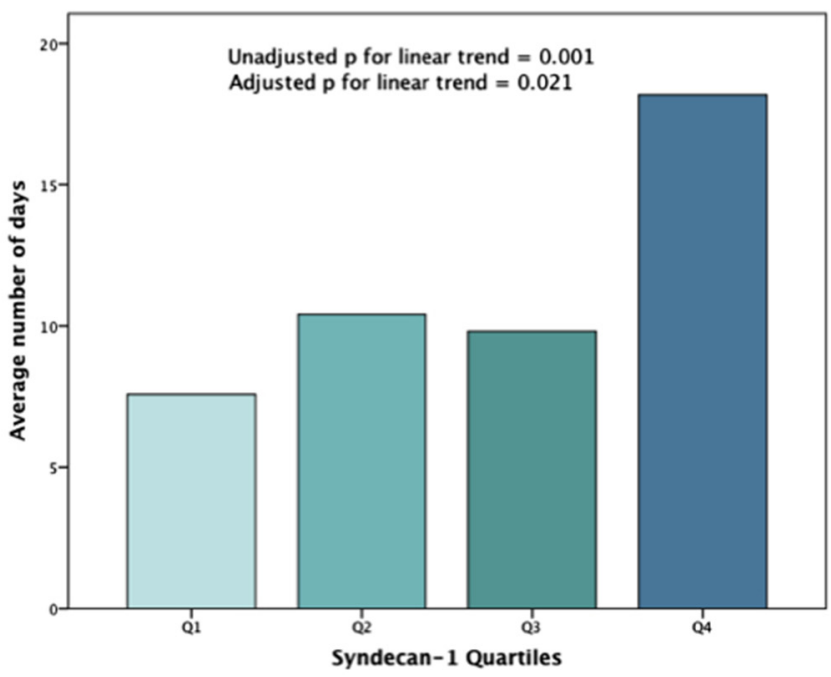

\section{Length of Mechanical ventilation}

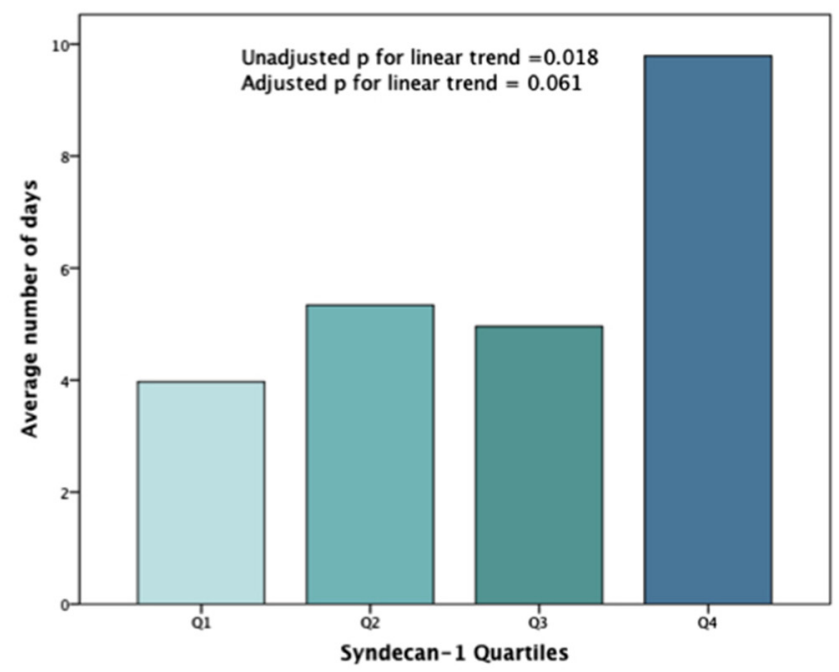

FIGURE 4. Linear association of postoperative syndecan-1 with longer length of hospital stay, ICU stay, and mechanical ventilation after adjustment for other prognostic factors. $I C U$, Intensive care unit.

not develop AKI. ${ }^{36-38}$ Moreover, in cardiac surgery, the lack of association between ICAM-1 increment and AKI can be explained, at least partly, by the observed reduction in their levels after CBP when compared with presurgery samples. Although adhesion molecules are known to have an important role in AKI pathophysiology, we speculate that postoperative systemic levels do not reflect their local expression in kidney tissue.
Although the use of syndecan-1 in clinical practice depends on further studies, it has some characteristics that could favor its widespread use: It is performed using an enzyme-linked immunosorbent assay, a usual laboratory technique; the assay cost was less than $\$ 12$ dollars in our research, and it can be even less expensive with widespread clinical use; and finally, it takes less than 2 hours to obtain the result. Early biomarkers associated with AKI can be 


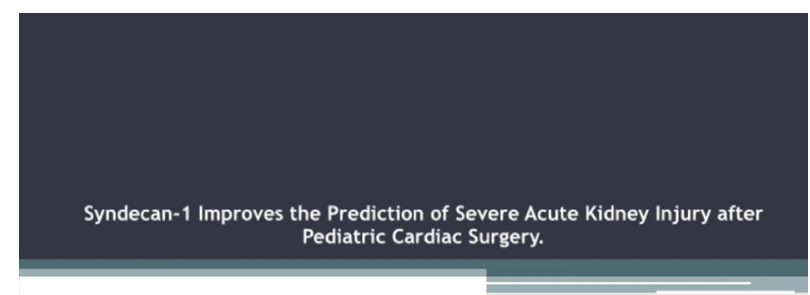

Candice Torres de Melo Bezerra Cavalcanteas, , MD, Klébia Magalhães Castelo Brancơ, M.D., Ph.D., Valdester Cavalcante Pinto Jünior', M.D., Gdayllon Cavalcante Meneses ${ }^{c}$, MsC, Fernanda Macedo Oliveira Neves, $s^{b}$, MsC, Nayana Maria Gomes de Souza, MsC, Kiarelle Lo
Alice Maria Costa Martins ${ }^{c}$, Ph.D., Alexandre Braga Libóriob, M.D., Ph.D.

VIDEO 1. Presentation of the main findings of the study. Video available at http://www.jtcvsonline.org/article/S0022-5223(16)30102-7/addons.

used in clinical practice to avoid exposing at-risk patients to further nephrotoxic drugs, for example. Although we do not have direct therapeutic agents available for the treatment of $\mathrm{AKI}$ at the present time, there are ongoing studies exploring potential future therapies. Biomarkers may be useful in selecting patients who might better respond to treatment modalities for AKI. Finally, we believe the next challenge for syndecan-1 and other AKI biomarkers is to test their ability to direct therapeutic intervention or other types of clinical management.

\section{Study Limitations}

Our study has several limitations, but at least 2 must be discussed: First, it was performed at a single center. Although our severe AKI rate was close to that described in the cited studies, our dialysis and mortality rates were 3- and 5-fold higher than those in developed countries, ${ }^{34}$ respectively. However, our rates were similar to those described in other developing countries. ${ }^{39,40}$ We suggest that other variables, such as delayed diagnosis of congenital heart disease and difficult access to specialist care, can contribute to such high mortality.

Second, we measured syndecan-1 at only 1 time point, early in the postoperative period. Bruegger and colleagues ${ }^{30}$ have demonstrated that there is a greater increment in plasma syndecan-1 level soon after CPB and that these levels remain stable up to patient admission in the ICU. They also showed that the mean value of preoperative syndecan-1 in children with congenital heart disease was $37.4 \mathrm{ng} / \mathrm{mL}$, a value 3 -fold lower than our measurement in the postoperative period. ${ }^{30}$ Because of this greater increment in postoperative value, it is unlikely that the preoperative syndecan-1 level can have a significant impact on our findings.

\section{CONCLUSIONS}

Early postoperative syndecan-1 level is a useful marker to predict severe AKI development after cardiac surgery in children (Video 1). Adding postoperative syndecan-1, even when using a clinical model that already incorporates variables from RAI, results in significant improvement in the capacity to predict severe AKI.

\section{Conflict of Interest Statement}

Authors have nothing to disclose with regard to commercial support.

\section{References}

1. Thiele RH, Isbell JM, Rosner MH. AKI associated with cardiac surgery. Clin J Am Soc Nephrol. 2015;10:500-14.

2. Esch JJ, Salvin JM, Thiagarajan RR, Del Nido PJ, Rajagopal SK. Acute kidney injury after Fontan completion: risk factors and outcomes. J Thorac Cardiovasc Surg. 2015;150:190-7.

3. Li S, Krawczeski CD, Zappitelli M, Devarajan P, Thiessen-Philbrook H, Coca SG, et al. Incidence, risk factors, and outcomes of acute kidney injury after pediatric cardiac surgery: a prospective multicenter study. Crit Care Med. 2011; 39:1493-9.

4. Alkandari O, Eddington KA, Hyder A, Gauvin F, Ducruet T, Gottesman R, et al Acute kidney injury is an independent risk factor for pediatric intensive care unit mortality, longer length of stay and prolonged mechanical ventilation in critically ill children: a two-center retrospective cohort study. Crit Care. 2011;15:R146.

5. Moran SM, Myers BD. Course of acute renal failure studied by a model of creatinine kinetics. Kidney Int. 1985;27:928-37.

6. Koyner JL, Parikh CR. Clinical utility of biomarkers of AKI in cardiac surgery and critical illness. Clin J Am Soc Nephrol. 2013;8:1034-42.

7. Verma SK, Molitoris BA. Renal endothelial injury and microvascular dysfunction in acute kidney injury. Semin Nephrol. 2015;35:96-107.

8. Alphonsus CS, Rodseth RN. The endothelial glycocalyx: a review of the vascular barrier. Anaesthesia. 2014;69:777-84.

9. Nieuwdorp M, Meuwese MC, Vink H, Hoekstra JBL, Kastelein JJP, Stroes ESG The endothelial glycocalyx: a potential barrier between health and vascular disease. Curr Opin Lipidol. 2005;16:507-11.

10. Schwartz GJ, Muñoz A, Schneider MF, Mak RH, Kaskel F, Warady BA, et al New equations to estimate GFR in children with CKD. J Am Soc Nephrol. 2009;20:629-37.

11. Piepsz A, Tondeur M, Ham H. Revisiting normal (51)Cr-ethylenediaminetetraacetic acid clearance values in children. Eur J Nucl Med Mol Imaging. 2006; 33:1477-82.

12. Jenkins KJ, Gauvreau K, Newburger JW, Spray TL, Moller JH, Iezzoni LI Consensus-based method for risk adjustment for surgery for congenital heart disease. J Thorac Cardiovasc Surg. 2002;123:110-8.

13. Haque IU, Zaritsky AL. Analysis of the evidence for the lower limit of systolic and mean arterial pressure in children. Pediatr Crit Care Med. 2007;8:138-44.

14. Gaies MG, Gurney JG, Yen AH, Napoli ML, Gajarski RJ, Ohye RG. Vasoactiveinotropic score as a predictor of morbidity and mortality in infants after cardiopulmonary bypass. Pediatr Crit Care Med. 2010;11:234-8.

15. Boeddeker SJ, Baston-Buest DM, Altergot-Ahmad O, Kruessel JS, Hess AP. Syndecan-1 knockdown in endometrial epithelial cells alters their apoptotic protein profile and enhances the inducibility of apoptosis. Mol Hum Reprod. 2014; 20:567-78.

16. KDIGO Board Members. Kidney Int Suppl (2011). 2012;2:3.

17. Agresti A. Categorical Data Analysis. Hoboken, NJ: Wiley; 2013.

18. Basu RK, Zappitelli M, Brunner L, Wang Y, Gajarski RJ, Ohye RG, et al. Derivation and validation of the renal angina index to improve the prediction of acute kidney injury in critically ill children. Kidney Int. 2014;85:659-67.

19. Moons KG, Donders AR, Steyerberg EW, Harrell FE. Penalized maximum likelihood estimation to directly adjust diagnostic and prognostic prediction models for overoptimism: a clinical example. J Clin Epidemiol. 2004;57:1262-70.

20. DeLong ER, DeLong DM, Clarke-Pearson DL. Comparing the areas under two or more correlated receiver operating characteristic curves: a nonparametric approach. Biometrics. 1988;44:837-45.

21. Pencina MJ, D'Agostino RB, Steyerberg EW. Extensions of net reclassification improvement calculations to measure usefulness of new biomarkers. Stat Med. 2011;30:11-21.

22. Cook NR. Statistical evaluation of prognostic versus diagnostic models: beyond the ROC curve. Clin Chem. 2008;54:17-23. 
23. Parikh CR, Devarajan P, Zappitelli M, Sint K, Thiessen-Philbrook H, Li S, et al. Postoperative biomarkers predict acute kidney injury and poor outcomes after pediatric cardiac surgery. J Am Soc Nephrol. 2011;22:1737-47.

24. Rehm M, Bruegger D, Christ F, Conzen P, Thiel M, Jacob M, et al. Shedding of the endothelial glycocalyx in patients undergoing major vascular surgery with global and regional ischemia. Circulation. 2007;116:1896-906.

25. Svennevig K, Hoel T, Thiara A, Kolset S, Castelheim A, Mollnes T, et al. Syndecan-1 plasma levels during coronary artery bypass surgery with and without cardiopulmonary bypass. Perfusion. 2008;23:165-71.

26. Liu K-L, Lee K-T, Chang C-H, Chen Y-C, Lin S-M, Chu P-H. Elevated plasma thrombomodulin and angiopoietin-2 predict the development of acute kidney injury in patients with acute myocardial infarction. Crit Care. 2014;18:R100.

27. Bucholz EM, Whitlock RP, Zappitelli M, Devarajan P, Eikelboom J, Garg AX, et al. Cardiac biomarkers and acute kidney injury after cardiac surgery. Pediatrics. 2015;135:e945-56.

28. de Neves FM, Meneses GC, Sousa NE, Menezes RR, Parahyba MC, Martins AM, et al. Syndecan-1 in acute decompensated heart failure: association with renal function and mortality. Circ J. 2015;79:1511-9.

29. Libório AB, Braz MBM, Seguro AC, Meneses GC, de Neves FMO, Pedrosa DC, et al. Endothelial glycocalyx damage is associated with leptospirosis acute kidney injury. Am J Trop Med Hyg. 2015;92:611-6.

30. Bruegger D, Brettner F, Rossberg I, Nussbaum C, Kowavski C, Januszewska K, et al. Acute degradation of the endothelial glycocalyx in infants undergoing cardiac surgical procedures. Ann Thorac Surg. 2015;99:926-31.

31. Padberg J-S, Wiesinger A, di Marco GS, Reuter S, Grabner A, Kentrup D, et al. Damage of the endothelial glycocalyx in chronic kidney disease. Atherosclerosis. 2014;234:335-43.

32. Zappitelli M, Coca SG, Garg AX, Krawczeski CD, Thiessen Heather P, Sint K, et al. The association of albumin/creatinine ratio with postoperative AKI in children undergoing cardiac surgery. Clin J Am Soc Nephrol. 2012;7:1761-9.
33. Parikh CR, Thiessen-Philbrook H, Garg AX, Kadiyala D, Shlipak MG, Koyner JL, et al. Performance of kidney injury molecule-1 and liver fatty acidbinding protein and combined biomarkers of AKI after cardiac surgery. Clin J Am Soc Nephrol. 2013;8:1079-88.

34. Zappitelli M, Krawczeski CD, Devarajan P, Wang Z, Sint K, ThiessenPhilbrook H, et al. Early postoperative serum cystatin $\mathrm{C}$ predicts severe acute kidney injury following pediatric cardiac surgery. Kidney Int. 2011; 80:655-62.

35. Zhou F, Luo Q, Wang L, Han L. Diagnostic value of neutrophil gelatinase-associated lipocalin for early diagnosis of cardiac surgeryassociated acute kidney injury: a meta-analysis. Eur $J$ Cardiothorac Surg. 2016;49:746-55.

36. Powell TC, Powell SL, Allen BK, Griffin RL, Warnock DG, Wang HE. Association of inflammatory and endothelial cell activation biomarkers with acute kidney injury after sepsis. Springerplus. 2014;3:207.

37. Boldt J, Osmer C, Linke LC, Dapper F, Hempelmann G. Circulating adhesion molecules in pediatric cardiac surgery. Anesth Analg. 1995;81:1129-35.

38. Le Dorze M, Legrand M, Payen D, Ince C. The role of the microcirculation in acute kidney injury. Curr Opin Crit Care. 2009;15:503-8.

39. Larrazabal LA, Jenkins KJ, Gauvreau K, Vida VL, Benavidez OJ, Gaitán GA, et al. Improvement in congenital heart surgery in a developing country: the Guatemalan experience. Circulation. 2007;116:1882-7.

40. Ekure EN, Esezobor CI, Sridhar A, Vasudevan J, Subramanyan R, Cherian KM. Cardiac surgery-associated acute kidney injury in a developing country: prevalence, risk factors and outcome. Saudi J Kidney Dis Transpl. 2015;26:489-96.

Key Words: acute kidney injury, syndecan-1, cardiac surgery 

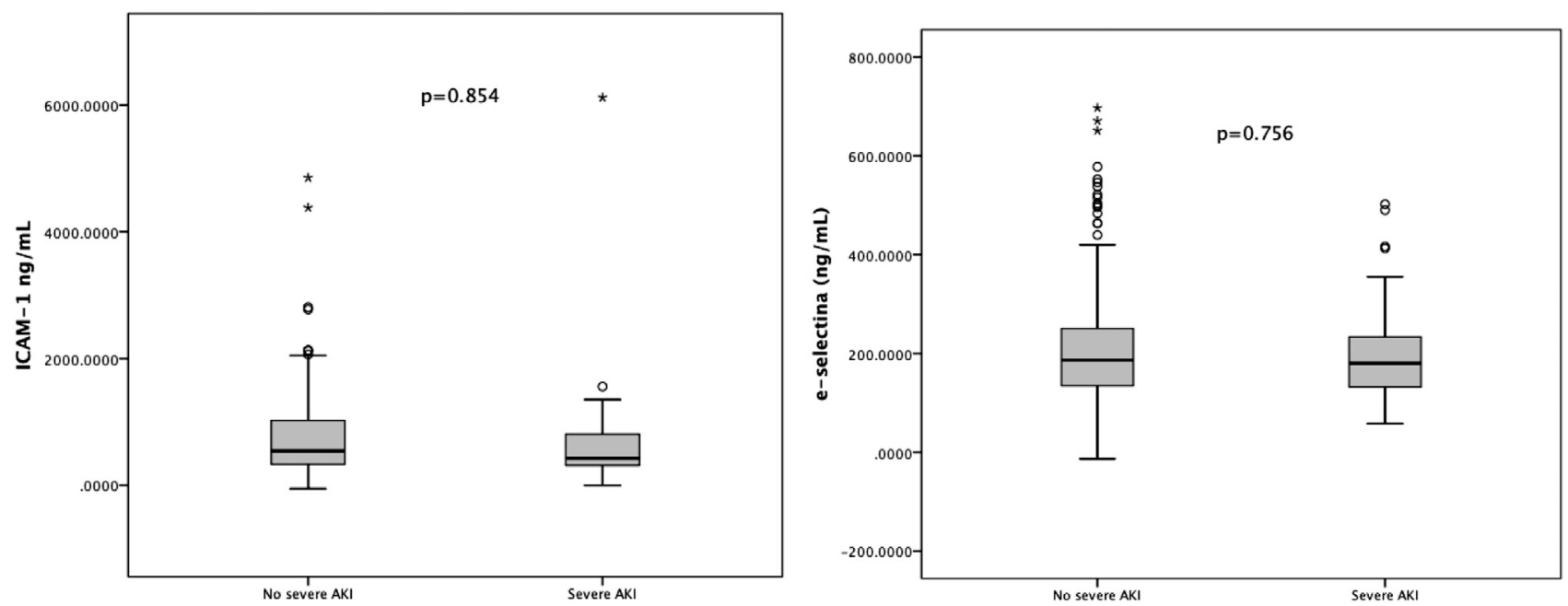

FIGURE E1. ICAM-1 and e-selectin in children with or without severe AKI. AKI, Acute kidney injury; ICAM-1, intercellular adhesion molecule-1.

TABLE E1. Association of postoperative syndecan-1 and severe acute kidney injury in children aged more than 30 days and submitted to Risk Adjustment in Congenital Heart Surgery-1 2 or more surgeries

\begin{tabular}{lcc}
\hline & \multicolumn{2}{c}{ Severe AKI* } \\
\cline { 2 - 3 } Syndecan quartiles (cutoffs in ng/mL) & Crude OR (95\% CI) & Adjusted OR full $\dagger$ (95\% CI) \\
\hline Q1 $(<17.0)$ & 1 (reference) & 1 (reference) \\
Q2 $(17.1-46.7)$ & $0.72(0.11-4.50)$ & $0.83(0.12-5.63)$ \\
Q3 (47.4-93.1) & $1.91(0.45-8.12)$ & $1.90(0.41-8.88)$ \\
Q4 (>96.3) & $8.00(2.17-29.44)$ & $6.64(1.69-26.03)$ \\
\hline
\end{tabular}

$A K I$, Acute kidney injury; $O R$, odds ratio; $C I$, confidence interval. *Severe AKI is defined as the need for dialysis or a 2-fold increase in serum creatinine during hospitalization. $\dagger$ Adjusted for age (per year), gender, CPB time $120 \mathrm{~min}$, RACHS-1 $\geq 3$, preoperative eGFR percentile, decrease in estimated creatinine clearance from baseline on the first postoperative day, and increase in percent ICU fluid overload on the first postoperative day.

TABLE E2. Diagnostic performance and categoric net reclassification improvement of clinical model adding or not adding postoperative biomarker in children aged more than 30 days and submitted to Risk Adjustment in Congenital Heart Surgery-1 2 or more surgeries

\begin{tabular}{|c|c|c|c|c|c|}
\hline & \multicolumn{5}{|c|}{ Severe AKI } \\
\hline & AUC-ROC (SEM) & $P$ for comparison & Nonevent NRI (SEM) & Event NRI (SEM) & Total NRI \\
\hline Plasma NGAL (ng/mL) & $0.69(0.06)$ & - & - & - & - \\
\hline Plasma syndecan-1 & $0.81(0.05)$ & - & - & - & - \\
\hline Clinical model & $0.79(0.05)$ & - & - & - & - \\
\hline Clinical model + NGAL & $0.80(0.04)$ & $.340^{*}$ & $0.01(0.01)^{*}$ & $0.03(0.03)^{*}$ & $0.04 *$ \\
\hline Clinical model + syndecan- 1 & $0.85(0.04)$ & $<.05 \dagger$ & $0.06(0.02)^{*}$ & $0.14(0.06)^{*}$ & $0.20 *$ \\
\hline Clinical model + NGAL + syndecan- 1 & $0.86(0.04)$ & $.308 \ddagger$ & $0.01(0.01) \ddagger$ & $0.07(0.05) \ddagger$ & $0.08 \ddagger$ \\
\hline
\end{tabular}

TABLE E3. Association of postoperative syndecan-1 and severe acute kidney injury in children receiving aortic crossclamping

\begin{tabular}{lcc}
\hline & \multicolumn{2}{c}{ Severe AKI* } \\
\cline { 2 - 3 } Syndecan quartiles (cutoffs in $\mathbf{~ n g / m L ) ~}$ & Crude OR (95\% CI) & Adjusted OR full $\nmid$ (95\% $\mathbf{~ C I ) ~}$ \\
\hline Q1 $(<17.0)$ & 1 (reference) & 1 (reference) \\
Q2 $(17.1-46.7)$ & $0.72(0.11-4.50)$ & $0.95(0.18-5.44)$ \\
Q3 (47.4-93.1) & $1.91(0.45-8.12)$ & $1.78(0.39-7.54)$ \\
Q4 $(>96.3)$ & $8.00(2.17-29.44)$ & $5.30(1.76-16.28)$ \\
\hline
\end{tabular}

$A K I$, Acute kidney injury; $O R$, odds ratio; $C I$, confidence interval. *Severe AKI is defined as the need for dialysis or a 2-fold increase in serum creatinine during hospitalization. $\dagger$ Adjusted for age (per year), gender, CPB time $120 \mathrm{~min}$, RACHS-1 $\geq 3$, preoperative eGFR percentile, decrease in estimated creatinine clearance from baseline on the first postoperative day, and increase in percent ICU fluid overload on the first postoperative day. 
TABLE E4. Diagnostic performance and categoric net reclassification improvement of clinical model adding or not adding postoperative biomarker in children receiving aortic crossclamping

\begin{tabular}{|c|c|c|c|c|c|}
\hline & \multicolumn{5}{|c|}{ Severe AKI } \\
\hline & AUC-ROC (SEM) & $P$ for comparison & Nonevent NRI (SEM) & Event NRI (SEM) & Total NRI \\
\hline Plasma NGAL (ng/mL) & $0.59(0.06)$ & - & - & - & - \\
\hline Plasma syndecan-1 & $0.78(0.05)$ & - & - & - & - \\
\hline Clinical model & $0.77(0.04)$ & - & - & - & - \\
\hline Clinical model + NGAL & $0.78(0.05)$ & $.590^{*}$ & $0.01(0.01)^{*}$ & $0.02(0.01)^{*}$ & $0.03^{*}$ \\
\hline Clinical model + syndecan-1 & $0.83(0.04)$ & $<.05 \dagger$ & $0.06(0.02)^{*}$ & $0.15(0.06)^{*}$ & $0.21^{*}$ \\
\hline Clinical model + NGAL + Syndecan-1 & $0.83(0.04)$ & $.610 \ddagger$ & $0.01(0.01) \ddagger$ & $0.04(0.03) \ddagger$ & $0.05 \ddagger$ \\
\hline
\end{tabular}

